Escuela de Ciencias Sociales y Humanidades, UNED, Costa Rica https://revistas.uned.ac.cr/index.php/espiga ISSN: 1409-4002 • e-ISSN: 2215-454X

\title{
La democratización de los contratos de conservación y mantenimiento de la red vial nacional: Un caso fallido en Costa Rica
}

\section{The Democratization of Contracts for the Conservation and Maintenance of the National Road Network: A Failed Case in Costa Rica}

La démocratisation des contrats de conservation et entretien du réseau routier national: un cas d'échec au Costa Rica

Jorge Alberto Vásquez-Rodríguez *

https://orcid.org/0000-0001-7094-3539

Recibido: 4 de julio de 2021 • Aceptado: 22 de setiembre de 2021

* Bachillerato, licenciatura y Magister Scientiae en Administración Pública, Universidad de Costa Rica (UCR), de Costa Rica. Licenciatura en Administración de Empresas con énfasis en Banca y Finanzas, Universidad Estatal a Distancia (UNED), de Costa Rica. Licenciatura en Estudios Latinoamericanos, Universidad Nacional (UNA), de Costa Rica. Magister Scientiae en Sociología, UCR. Doctorado Académico en Gobierno y Políticas Públicas, UCR. Tutor e investigador de la Escuela de Ciencias de la Administración, de la UNED. Correo: jvasquez@uned.ac.cr 


\section{Resumen}

El objetivo de esta investigación, en la que se utilizaron métodos cualitativos y cuantitativos de investigación, así como métodos cualitativos de análisis, fue determinar las causas por las cuales no fue posible, mediante la Licitación Pública No. 2014LN000016-0CV00 MR-I: Mantenimiento Rutinario sin Maquinaria Especializada de la Red Vial Nacional Pavimentada, contratar únicamente a pequeñas y medianas empresas (Pymes) para la limpieza de hierbas y basura de la red vial nacional de Costa Rica, como pretendía la administración Chinchilla Miranda (2010-2014). El propósito del espíritu de la licitación se logró parcialmente, en tanto, las grandes empresas, dentro del marco de legalidad del país y aprovechando su infraestructura, participaron en el concurso, acaparando parte del contrato de mantenimiento vial sin maquinaria especializada de las 22 zonas en que se compone el país, para efectos de conservación y mantenimiento. Las causas principales de lo anterior fueron un marco institucional que no facilitó la contratación de las Mypmes y la licitación perdió su patrocinador en el Poder Ejecutivo. Con el cambio de gobierno, la burocracia encargada de la ejecución final de la contratación no se encontraba motivada para llevar a cabo la licitación y los micro, pequeños y medianos empresarios no contaban con los conocimientos ni la infraestructura adecuada para participar en el concurso público (licitación), aunado a los escasos recursos financieros; además de que los trabajadores que prestan el servicio directamente (peones) fueron invisibilizados de todo el proceso.

Palabras clave: Compras públicas, contrataciones, gestión pública, reforma del Estado.

\section{Abstract}

The objective of this research, in which qualitative and quantitative research methods were used, as well as qualitative methods of analysis, was to determine the causes as to why it was not possible, through Public Tender No. 2014LN-000016-0CV00 MR-I: Routine Maintenance without Specialized Machinery of the National Paved Road Network, to hire only small and medium-sized enterprises(SMEs) for the cleaning of weeds and other waste from the national road network of Costa Rica, as intended by the Chinchilla Miranda administration (2010-2014). The purpose of the spirit of the tender was partially achieved, while large companies, within the framework of legality of the country and taking advantage of its infrastructure, participated in the contest, monopolizing part of the road maintenance contract without specialized machinery of the 22 areas in which the country is composed, for conservation and maintenance purposes. The main causes of this were an institutional framework that did not facilitate the contracting of the SMEs and the tender lost its sponsor in the Executive Branch. With the change of government, the bureaucracy in charge of the final execution of the contract was not motivated to carry out the tender and the micro, small and medium entrepreneurs did not have the knowledge or the adequate infrastructure to participate in the public tender (tender), coupled with their scarce financial resources; in addition, the workers who provide the service directly (laborers) were made invisible from the whole process.

Keywords: Public contracts, procurement, public management, state reform.

\section{Résumé}

Le but de cette recherche a été déterminer les causes par lesquelles n'a pas été possible à travers I'adjudication publique № 2014LN-000016-0CV00 MR-I: Entretien de Routine sans Machines Spécialisées du Réseau Routier National Pavé, embauche unique de 
petites et moyennes entreprises (PME) pour le nettoyage des herbes et des déchets du réseau routier national du Costa Rica dans la gestion Chinchilla-Miranda (2010-2014). Cette étude a été mené moyennant des méthodes quantitatives et qualitatives, notamment des méthodes qualitatives pour l'analyse. L'intention de l'adjudication a été atteinte partiellement parce que les grandes entreprises ont participé dans le concours en profitant du cadre juridique du pays et de leur infrastructure, en accaparant une grande partie de l'adjudication d'entretien routier sans machines spécialisées des 22 zones du pays. Les principales causes de cette monopolisation ont été le cadre institutionnel qui n'a pas favorisé l'embauche des PME, ainsi que la perte de l'appui qui a eu l'adjudication de la part du Pouvoir Exécutif. Lors du changement de gouvernement, l'administration responsable de l'exécution finale de l'embauche n'a pas été motivée pour mener à bien l'adjudication, tandis que les petits et moyennes entrepreneurs n'avaient ni les connaissances ni l'infrastructure adéquate pour participer au concours public (adjudication). À cela s'ajoute les ressources limitées et l'invisibilisation des travailleurs (ouvriers) qui fournissent le service directement pendant tous le procès.

Mots-clés: Achats publics, adjudications, gestion publique, réforme de l'État. 


\section{Introducción}

En la administración Chinchilla Miranda (2010-2014) se formuló la «Política Pública de Fomento a las Pyme y al emprendedurismo», mientras que en la segunda mitad de esa administración, como parte del Programa Nacional de Compras Públicas, se gestionó la Licitación Pública No. 2014LN-000016-0CV00 MR-I: Mantenimiento Rutinario sin Maquinaria Especializada de la Red Vial Nacional Pavimentada, con el propósito de brindar acceso a las compras públicas del Estado, que habían permanecido cautivas de las grandes empresas de la construcción, conservación y mantenimiento vial nacional en el país.

En la primera mitad de la administración Solís Rivera (2014-2018), se adjudicaron a los oferentes 20 de las 22 zonas en que se había dividido el país, para efectos de brindar mantenimiento de limpieza a las vías nacionales (limpieza de hierbas y basura de la red vial nacional), lo que popularmente se denomina «Chapea».

El proceso de adjudicación tuvo que superar una serie de cuestionamientos y apelaciones relacionadas con la naturaleza de las micro, pequeñas y medianas empresas (Mipymes), que participaron en la licitación, algunas de ellas vinculadas con las grandes empresas nacionales que tienen cautivas las compras públicas en el ámbito de la construcción, conservación y mantenimiento vial nacional.

El proceso de contratación tardó casi cuatro años, con lo cual se logró, en forma parcial, el objetivo inicial de democratizar las compras públicas del ámbito mencionado, ya que se le brindó acceso parcial a las compras públicas a las Mipymes, pero en el proceso se filtraron representantes de los grandes empresarios, además de que no fue posible concretar un contrato para 2 de las 22 zonas del país.

En todo el proceso de contratación, desde su génesis hasta su adjudicación, fueron invisibilizados los trabajadores que directamente prestan los servicios de limpieza (peones).

El caso anterior, fue el objeto de la investigación que se expone en el presente artículo, principalmente bajo la perspectiva teórica de la política pública, el neoinstitucionalismo, la burocracia weberiana, el homo economicus, el homo bureaucratic y el estilo nacional de desarrollo. 


\section{Aspectos teóricos}

De conformidad con Subirats et all ${ }^{1}$, la política pública es una repuesta, por parte de las autoridades públicas, a un problema social que ha sido incorporado a la agenda política.

El objetivo de la política pública es la resolución de los problemas colectivos, reconocidos como tales por las autoridades públicas y, por tanto, considerado un problema ${ }^{2}$ público ${ }^{3}$. La política pública hace referencia a las interacciones, alianzas y conflictos, en un marco institucional específico, entre los diferentes actores públicos y privados, para resolver un problema colectivo que se requiere de una acción concreta. Debe entenderse como política pública a:

Una serie de decisiones o de acciones, intencionalmente coherentes, tomadas por diferentes actores, públicos y a veces no públicos-cuyos recursos, nexos institucionales e intereses varían a fin de resolver de manera puntual un problema políticamente definido como colectivo. Este conjunto de decisiones y acciones da lugar a actos formales, con un grado de obligatoriedad variable, tendentes a modificar la conducta de grupos sociales que, se supone, originan el problema colectivo a resolver (grupos objetivos), en el interés de grupos sociales que padecen los efectos negativos del problema en cuestión (beneficiarios finales). ${ }^{4}$

Del anterior concepto se derivan los siguientes aspectos clave:

i. La existencia de un problema público y sus causas (hipótesis causal).

ii. Una posible solución al problema público (hipótesis de solución).

iii. Actores públicos y privados que intervienen en el problema público y en su solución.

iv. El reconocimiento de que los actores tienen intereses y recursos, para satisfacer esos intereses.

v. La existencia de instituciones formales e informales.

vi. El requerimiento de una cierta formalidad, como una norma (ley, reglamento) emitida por una autoridad pública legitimidada para tal efecto.

\footnotetext{
1 Joan Subirats, Peter Knoepfel, Corinne Larrue y Fréderic Varones, Análisis y gestión de políticas públicas (España: Editorial Ariel, 2008).

2 Ibíd.

3 Jorge Vásquez Rodríguez, «La Política Pública de la Infraestructura Vial Nacional en Costa Rica (1998-

2014)», tesis doctoral, presentada en el Programa de Doctorado en Gobierno y Políticas Públicas, Universidad de Costa Rica, 2019.

${ }^{4}$ Subirats et al., «Análisis y gestión..., 38.
} 
Al analizar esos elementos clave que constituyen la política pública, se evidencia que se fundamenta en el neonstitucionalismo basado en actores, ya que retoma la importancia de las reglas, formales o no, así como de los actores en relación con ellas, «incrustrado o «atrapado» en las reglas institucionales formales e informales, adopta conductas políticas que se adecuan a los valores y a las expectativas que las citadas reglas proponen, pero al mismo tiempo las modifica de manera incremental mediante sus propias decisiones y acciones 5 .

Los resultados de una política no son el producto exclusivo de las reglas institucionales, sino de la actuación de los actores en ese marco institucional6; actores e instituciones ${ }^{7}$ se influyen mutualmente ${ }^{8}$.

La política pública ocurre dentro de un determinado estilo nacional de desarrollo, en donde la política es influenciada por el estilo o podría suceder que esta influya también ${ }^{9}$ en él ${ }^{10}$. Rovira ${ }^{11}$ indica que:

La noción de estilo de desarrollo lo que busca es describir, e igualmente aprehender sucintamente y de un modo comprensivo, el movimiento concreto que sigue una sociedad particular, no un tipo genérico de ella, durante el lapso histórico. No se trata tan solo de los componentes socioeconómicos, sino también de los políticos, de los culturales y de los medioambientales. Más aún, en la aprehensión de un estilo de desarrollo los factores políticos, en especial los factores políticos internos, desempeñan un papel crucial, de primer orden.

De conformidad con Graciarena ${ }^{12}$ y Rovira ${ }^{13}$, el estilo nacional de desarrollo busca la comprensión y rumbo de los componentes socioeconómicos, culturales, medioambientales y políticas, de una determinada sociedad, pero el preponderante es el componente político por ser el decisor.

El estilo nacional de desarrollo es un producto históricamente elaborado que ha surgido de la interacción entre actores políticos, sus visiones, intereses y estrategias, en el cual prevalece la posición de los actores que poseen suficientes recursos de poder diferenciados claramente en la escena política ${ }^{14}$.

\footnotetext{
5 lbíd, 201.

6 Ibíd.

${ }^{7}$ Cristina Zurbriggen, «Las redes de políticas públicas. Una revisión teórica», Uruguay: Instituto de Ciencia Política N. 1, 2003, 1-15.

8 Jorge Vásquez Rodríguez, «La Compleja Institucionalidad de la Infraestructura Vial Nacional en Costa Rica, 1998-2014», Revista Nacional de Administración 8, n. ${ }^{\circ} 1$ (2017): 100-117, https://doi.org/10.22458/ rna.v8i1.1180

9 Vásquez Rodríguez, «La Política Pública....».

10 Jorge Rovira Mas, «Costa Rica: El actual estilo nacional de desarrollo y sus principales tendencias», en Róger Churnside y Eduardo Lizano (Compiladores y editores), Sociedad para el Avance de la Socioeconomía (SASE). Selecciones de la XX Conferencia Costa Rica 2008. San José: Academia de Centroamérica, 2009,163-178, http://www.hacienda.go.cr/centro/datos/Libro/

Sociedad\%20para\%20el\%20avance\%20de\%20la\%20socioeconom\%C3\%ADa.pdf

11 lbíd., 311.

12 Jorge Graciarena, citado en Jorge Vásquez Rodríguez, «La Política Pública de.... ».

13 Rovira Mas, 2009

14 Ibíd., 312.
} 
Rovira distingue cinco dimensiones con el propósito de aprehender sucintamente el estilo, que son:

i. Los factores condicionantes, especialmente aquellos impuestos desde el exterior, en particular la de los actores internacionales con peso suficiente para imponer o influir decisivamente en las decisiones políticas nacionales.

ii. Los factores determinantes, que son generalmente impuestas 0 influenciadas por actores internos, aunque también pueden considerarse los actores externos.

iii. La dirección que sigue el estilo.

iv. El ritmo que le es propio al estilo.

v. Las características que va adquiriendo paulatinamente la sociedad como resultado de los factores anteriores.

En el caso de Costa Rica, Rovira indica que el actual estilo nacional de desarrollo, de apertura comercial con un fuerte protagonismo del mercado en deterioro del Estado, inicia en la administración Monge Álvarez (1982-1986); a partir de ese momento, el país vendría «en forma paulatina, pero sostenida a seguir los postulados del Consenso de Washington, de los Chicago Boy`s y los Harvard Boy`s, apoyados en el territorio nación» ${ }^{15}$.

El actual estilo nacional de desarrollo se caracteriza por la desregulación, la privatización, la apertura de los monopolios estatales, la internacionalización de la economía, la paulatina desgravación de los aranceles a los productos internacionales, la normalización y disminución de subsidios, la prevalencia del mercado sobre el Estado.

Los pilares que sustentan la estrategia de reformas económicas actuales son:

i. Liberalización del comercio exterior y el retorno a las exportaciones como el motor de la economía.

ii. Reforma del sistema financiero.

iii. Reforma funcional del papel del Estado y su reducción institucional.

iv. Reforma laboral.

El Estado costarricense se ha reformado constantemente para adaptarse al llamado Estado mínimo, impulsado por el Consenso de Washington, y posteriormente al Estado necesario, asociado al post-Consenso de Washington,

15 Jorge Vásquez Rodríguez, «La Corporatización Empresarial de las Políticas Pública, el caso de Procomer», tesis de Maestría en Sociología, Universidad de Costa Rica, 2008, 216. 
que ocurre especialmente a partir de 1997, con el Banco ${ }^{16}$ Mundial17. El estilo nacional de desarrollo se encuentra determinado por actores clave, con recursos para tomar las decisiones que definen el mismo. De igual forma, las políticas públicas se formulan e implementan en un determinado estilo nacional de desarrollo, donde son importantes los actores, sus recursos, visiones e intereses, en tanto inciden en la formulación de la política y el marco institucional.

En esta urdimbre entre estilo y políticas públicas, las compras públicas han sido parte del proceso de transformación el Estado, en donde, en el nuevo escenario, las grandes empresas se han vuelto protagonistas, al acapararlas, en deterioro de las micro, pequeñas y medianas empresas.

Para distinguir y entender los intereses de los actores que se encuentran presentes en el estilo nacional de desarrollo y en las políticas públicas que ocurren en este, se hace referencia al homo economicus y al homo bureaucratic $^{18}$, el primero busca satisfacer su propio interés, por tanto sus acciones están orientadas a ese interés; y el segundo, de conformidad con Merton y Weber ${ }^{19}$, centra su interés en la hiperconformidad, desarrollada a partir del cargo o cargos que desempeña en la burocracia pública ${ }^{20}$, cuya permanencia en él se asocia al cumplimiento ciego de normas, alejado de la productividad, la calidad o la orientación a la ciudadanía.

\section{Aspectos metodológicos}

El periodo de estudio se extendió desde el 2012 hasta el 2016, debido a que en él se gestionó la Licitación Pública No. 2014LN-000016-0CV00 MR-I. «Mantenimiento Rutinario sin Maquinaria Especializada de la Red Vial Nacional Pavimentada», Licitación Pública No. 2014LN-000016-0CV00 MR-I, que constituye el caso de estudio ${ }^{21}$.

En la investigación se utilizaron métodos cualitativos y cuantitativos de investigación, así como métodos cualitativos de análisis. El método cualitativo de investigación utilizado fue la entrevista y la recopilación documental, apoyado por datos cuantitativos, vertidas en estadísticas22. La revisión documental consistió en la recopilación de documentación relacionada con las

\footnotetext{
16 Ibíd.

17 Rovira Mas, 2009

18 Vásquez Rodríguez, «La Política Pública...».

19 lbíd.

${ }^{20}$ Robert Márquez Blas, «Teorías clásicas de la burocracia capitalista: Hegel, Marx, Weber y Michells»,

Revista de Colaboración Jurídica, n. ${ }^{\circ} 76$ (1990): 77-89.

${ }^{21}$ Contraloría General de la República, expediente de Licitación Pública No. 2014LN-000016-0CV00 MR-I: Mantenimiento Rutinario Sin Maquinaria Especializada de la Red Vial Nacional Pavimentada. Contraloría General de la República, Costa Rica, 2015. Acceso: 21 de noviembre de 2019, https://cgrweb.cgr.go.cr/pr02/f?

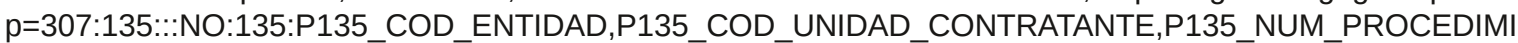
ENTO,P135_TIPO_PROCEDIMIENTO:2100042008,1000000415,2014LN-000016-0CV00,01

22 Fernando López Noguero, «El Análisis de Contenido Como Método de Investigación», Revista de Educación», 4 (2002): 167-179, Universidad de Huelva, 2002.
} 
compras públicas en Latinoamérica, en particular la utilización de estas como instrumento de política pública, asimismo se consideró las políticas públicas relacionados con el tema de investigación de las administraciones Chinchilla Miranda y Solís Rivera, el expediente de la Licitación Pública No. 2014LN000016-0CV00 MR-I y las noticias digitales relacionadas con dicho concurso público. En cuanto a las entrevistas, se realizaron a personas clave en el Ministerio de Economía, Industria y Comercio (MEIC) y el Consejo Nacional de Vialidad (Conavi).

Los métodos cualitativos de análisis utilizados fueron el de contenido, coyuntura y casos, que en conjunto con las teorías clave mencionadas (política pública, neoinstitucionalismo, burocracia weberiana, homo economicus, homo bureaucreactic, estilo nacional de desarrollo), permitieron el análisis de la información y el análisis del objeto de estudio.

En relación con el contenido, de conformidad con Berelson ${ }^{23}$ y López $^{24}$, se realiza una descripción objetiva, sistemática y cuantitativa. El método de coyuntura fue utilizado para contextualizar la política y extraer, de ese contexto, aspectos clave para la comprensión del fenómeno estudiado 25 . El método de casos fue adoptado en tanto el fenómeno en estudio es contemporáneo, poco estudiado y la frontera entre este y el contexto no se encuentra claramente delimitado ${ }^{26}$, en tanto las compras públicas en Costa Rica, relacionadas con la construcción, conservación y mantenimiento vial, se encuentran cautivas, por parte de un grupo de grandes empresas constructoras. Este mercado ha sido vedado o de difícil penetración a los micro, pequeños y medianos empresarios, y fue en ese contexto que precisamente se gestionó la contratación en mención con el propósito de hacer más extensivo las contrataciones de dicho sector a una mayor cantidad de ciudadanos.

La investigación pretende dar respuesta a la siguiente pregunta: ¿Por qué no fue posible democratizar en su totalidad las compras relacionadas con la conservación y mantenimiento vial sin maquinaria especializada (chapea) en el periodo 2012-2016, en Costa Rica?

\section{Compra pública como instrumento de política pública}

La transformación del Estado en América Latina, especialmente a partir de la década de 1980 y hasta la fecha, desde uno social a uno con rasgos neoliberales, ha permitido e impulsado, con mayor profundidad, el protagonismo de las compras públicas.

\footnotetext{
23 Berelson (1952), citado en Vásquez Rodríguez, «La Política Pública....».

${ }^{24}$ López (2002), citado en Vásquez Rodríguez, «La Política Pública....».

25 Helio Gallardo, Fundamentos de Formación Política. Análisis de Coyuntura (Costa Rica: Red Alforja Roja, 1990).

${ }^{26}$ Robert Yin, Applications of Case Study. Thousand Oaks (United States of America: Sage Publishing, 1994).
} 
En dicho periodo el Estado optó por contratar el suministro de bienes y servicios, en lugar de producirlos, lo que conllevó a que se redimensionara el empleo público, desprendiéndose de la necesidad de ocupar a grandes masas de trabajadores en la producción de esos bienes y servicios y, en su lugar, emplear a un reducido número de ellos, que laborarían en los procesos de contratación y administración de los contratos, con empresas privadas que suministrarían directamente los bienes y servicios ${ }^{27} 28$.

Este ascenso del protagonismo de las compras públicas tendría dos dimensiones, una interna y otra externa, ambas con un impacto en el empleo público, en la composición de los presupuestos públicos, la cantidad y calidad de los bienes y servicios brindados a la ciudadanía 2930.

Se redimensionaría entonces la esfera interna, contratando los servicios de apoyo requeridos para las operaciones sustantivas de los entes públicos, entre ellos los servicios de: seguridad, limpieza, correspondencia, diseño y mantenimiento de sistemas informáticos, entre otros.

Con la redimensión interna, entonces no fue necesario contar con grandes masas de empleados públicos, trabajando en puestos de seguridad, limpieza, diseño de sistemas informáticos, entre otros, sino un pequeño número de empleados a cargo de la contratación de esos servicios y la administración de los contratos correspondientes.

Pero, paulatinamente, las compras públicas se extenderían a la dimensión externa de los entes públicos, relacionados directamente con el suministro de los servicios y bienes que dichos entes debían suministrar al ciudadano.

Los servicios de salud, educación, conservación y mantenimiento de obras públicas, entre ellas las carreteras, entre otras, serían brindados directamente al ciudadano por empresas privadas, que para ese fin se contratarían.

El protagonismo de las compras públicas implicó que los presupuestos que antes se destinaban a sufragar los gastos de las grandes masas de empleados públicos, así como del mantenimiento de maquinarias, herramientas y edificaciones, y la compra de insumos, se destinará a las compras públicas, no necesariamente en las mismas cantidades.

Todo lo anterior llevó a que las compras públicas alcanzaran niveles considerables del Producto Interno Bruto (PIB), incluso del $15 \%$ de este -al menos en Latinoamérica- en el $2016^{31} 32$.

\footnotetext{
27 Vásquez Rodríguez, «La Política Pública...».

28 Nuria Cunill, «¿Qué ha pasado con lo público en los últimos 30 años? Balance y perspectivas», Revista del CLAD Reforma y Democracia, n. ${ }^{\circ} 52$ (2012): 5-7.

${ }^{29}$ Vásquez Rodríguez, «La Política Pública...».

30 Cunill, «¿Qué ha pasado...», 2012.

31 Vásquez Rodríguez, «La Compleja Institucionalidad...».

32 Yesenia Ledezma Rodríguez, «Un planteamiento de mecanismos para la evaluación de las ofertas que fomenten en las Pymes mayor accesibilidad de las compras de la administración pública en Costa Rica», tesis de Maestría en Gestión de la Innovación Tecnológica, Universidad Nacional, Costa Rica, 2015.
} 
Pese al gran mercado que representan las compras públicas, las micro, pequeñas y medianas empresas no han tenido acceso pleno a ellas, debido a que no cuentan con los recursos financieros ni los conocimientos para participar en los diferentes procesos ${ }^{33}$, por lo que en la administración Chinchilla Miranda existió la inquietud de democratizar dichas compras públicas.

En Brasil, Bolivia, Colombia, Ecuador, México, Paraguay, Uruguay y Venezuela 34 hay iniciativas con el propósito de fomentar la participación de las Pymes en las compras públicas. En algunos de estos países se establecen cuotas para las Pymes, como es el caso de Brasil, mientras que en otros países que se les adjudica, con precio mayor, si cumplen las condiciones cartelarias, como es el caso de Bolivia, hecho que para el caso particular de Costa Rica, podría violar los principios constitucionales en contratación administrativa.

\section{Marco normativo de las compras públicas}

El marco general de las compras públicas en Costa Rica lo constituye una serie de principios constitucionales emanados del artículo 182 de la Constitución Política de 1949, establecidos por la Sala Constitución del país, en 1998, mediante el voto 98 de ese año, por la Ley de Contratación Administrativa, N ${ }^{\circ}$ 7494, su reglamento y una serie de jurisprudencia judicial y administrativa, esta última de la Contraloría General de la República. Cabe mencionar que existe una regulación particular para el sector telecomunicaciones, de conformidad con la Ley de Fortalecimiento y Modernización de las Entidades Públicas del Sector Telecomunicaciones, Ley № 8660, publicada en el Alcance № 31 de La Gaceta № 156 de 13 de agosto de 2008.

Con la incorporación del gobierno digital en la gestión pública, el marco general anterior de compras públicas se complementó con la Ley de Certificados, Firmas Digitales y Documentos Electrónicos del 13 de octubre de 2005, el Decreto Ejecutivo № 35139-MP-Mideplan, que estableció la Comisión Interinstitucional de Gobierno Digital, el Decreto Ejecutivo No. 38830-H-MICITT que «Crea el Sistema Integrado de Compras Públicas como Plataforma Tecnológica de Uso Obligatorio de la Administración Central para la Tramitación de los Procedimientos de Contratación Administrativa SICOP» del 15 de enero de 2015.

\section{Sistema Integrado de Compras Públicas (Sicop)}

En la primera década del presente lustro, especialmente en la segunda administración Arias Sánchez (2006-2010), en el marco del Gobierno Digital y

\footnotetext{
33 Vásquez Rodríguez, «La Política Pública...».

34 Cegesti, Análisis de la realidad de acceso y participación de las Pymes en las compras públicas en Costa Rica, Programa ICT4GP, Ministerio Relaciones Exteriores Reino de los Países Bajos, Costa Rica, 2012, acceso: 12 de octubre de 2020, http://comprasresponsables.cegesti.org/articulos/articulo-25260213_informe_compras_publicas_PYME_CR.pdf
} 
posteriormente en las administraciones Chinchilla Miranda y Solís Rivera, como parte del Gobierno Abierto, se informatizarían las compras públicas, iniciando con Comprared 1, que sería sustituido por Comprared 2, el cual coexistiría con Mer-link, estos dos últimos sustituidos por el Sistema Integrado de Compras Públicas (Sicop).

Con el Sicop, se pretendió integrar todas las compras públicas del Estado costarricense en forma obligatoria, para la administración central, y voluntaria, para los demás entes públicos, incluyendo las municipalidades.

El Sicop, concordante con las premisas del Gobierno Digital, pretendió mejorar la eficiencia y eficacia de las compras públicas mediante el uso de las tecnologías de la información y, dentro del marco del Gobierno Abierto, aspiraba a democratizar las compras públicas en varios aspectos. Al estar visibles, las compras públicas, en un sistema informático de libre acceso a toda la ciudadanía, mediante una conexión a internet, permitiría a todo posible proveedor participar en los concursos de compras públicas y, a su vez, a la ciudadanía en general, monitorearlas desde su gestación y hasta la adjudicación, incluso en su ejecución del cumplimiento del contrato correspondiente.

Con el Sicop, se pretendió la mejora de la eficiencia y eficacia de las compras públicas y la democratización de estas últimas; aunque no se encontró ningún estudio que demuestre que esto último se haya logrado.

\section{Marco normativo de compras públicas para Mipymes}

Para el caso de las compras públicas realizadas a Mipymes, el marco normativo para su impulso es débil, ya que dichas empresas deben sujetarse, igual que cualquier otra, a las normas generales antes citadas, complementado con la normativa del Gobierno Digital, existiendo como única ventaja normativa, lo establecido en el artículo 20 de la Ley de Fortalecimiento de las Pequeñas y Medianas Empresas, Ley $\mathrm{N}^{\circ} 8262$ del 2 de mayo de 2002. Esta indica que, en igualdad de condiciones entre varias ofertas presentadas para un determinado concurso, se escogerá a la oferta de la Mipymes registrada en el MEIC.

Si bien en la segunda administración Arias Sánchez se aprobó el Reglamento Especial para la Promoción de las Pymes ${ }^{35}$ en las Compras de Bienes y Servicios de la Administración, Decreto Ejecutivo N 33305-MEIC-H, del 1 de setiembre, en esencia, este repite lo que el artículo 20 de la ley $\mathrm{N}^{\circ} 8262$ ya había mencionado y no aporta un valor agregado a la normativa ya existente, en tanto, únicamente hace referencia a aspectos que ya habían sido regulados para las compras públicas en general, sin hacer una diferencia sustantiva con las Mipymes, salvo la ya apuntada. En esencia, se puede resumir en igualdad de condiciones, incluyendo el precio, que se prefiera a la Pymes como un criterio de desempate.

35 La diferente normativa que rige las micro, pequeñas y medianas empresas (Mipymes) en Costa Rica, hacen mención a las pequeñas y medianas empresas (Pymes); sin embargo, debe entenderse que lo regulado para las Pymes se extiende a las microempresas. 
Cautiverio de las compras públicas relacionadas con obra vial nacional

Con la Ley de Creación del Conavi, №7798, en 1998, se reorientó la política de infraestructura vial nacional de conformidad con el estilo nacional de desarrollo que había imperado en el país desde la administración Monge Álvarez (19821986), en la cual el Estado dejó de construir, conservar y mantener la red vial nacional con su propia planilla, maquinaria, herramientas y materia prima para, en su lugar, mediante concurso público, contratar a empresas privadas para que realizaran esas labores.

Desde ese momento ha existido una serie de licitaciones públicas, las de mayor cuantía en el Estado de Costa Rica, relacionadas con la obra vial nacional, en las que menos de cinco empresas han acaparado las contrataciones relacionadas para dicha obra ${ }^{36}$.

De 1998 a 2016, mediante el Conavi (ente que fue creado para la contratación y administración de contratos viales), Costa Rica ha tenido presupuestos que oscilan entre los 20 mil millones de colones hasta 500 mil millones de colones por año, de los cuales, de conformidad con la Ley 7798 , se destina el $95 \%$ de ese presupuesto directamente a obra vial, quedando el $5 \%$ restante a gastos administrativos.

\section{Política pública}

En la administración Chinchilla Miranda, el MEIC formuló la «Política Pública de Fomento a las Pymes y al Emprendurismo», que estableció en el Área de Acceso a Mercados que tenía como objetivo el de «Promover el acceso a las Pyme a los diferentes canales de comercialización locales e internacionales» el Programa Nacional de Compras Públicas, que pretendía, incrementar la participación de las Pyme como proveedores del Estado mediante el fortalecimiento de instrumentos de acceso a los procesos de licitación pública y la concientización de las proveedurías institucionales para compras de los bienes y servicios brindados por las Pymes ${ }^{37}$. Al comparar las acciones de dicho programa con sus indicadores, se puede establecer que las acciones concretas que articularía la política serían las siguientes:

i. Capacitación a la Mipymes para que participaran en el proceso de compras públicas, la cual se llevaría a cabo mediante cursos impartidos por el Instituto Nacional de Aprendizaje (INA).

ii. El registro de las Mipymes ${ }^{38}$ como proveedores en el Estado.

iii. Capacitación a los funcionarios de las proveedurías de los entes públicos.

36 Vásquez Rodríguez, «La Política Pública...».

37 Contraloría General de la República, expediente de Licitación Pública...

38 Se menciona a las Pymes, pero es extensivo a las microempresas. 


\section{Problema público}

La política pública reconocía como problema público la falta de acceso de las Pymes a las compras públicas, al identificar las causas siguientes: públicas.

i. Falta de capacitación de los funcionarios de las Pymes en compras

ii. Falta de conciencia de las jefaturas y el resto de los funcionarios de las direcciones de proveeduría, encargadas de realizar las contrataciones.

iii. La identificación de la falta de competencias de las Pymes es causa certera del problema público 39 40, ya que dichas empresas no contaban con los conocimientos necesarios para a) inscribirse en los registros de proveedores, b) preparar las ofertas y c) presentar los recursos administrativos del proceso de contratación.

La concientización de las jefaturas de las proveedurías y el resto de su personal, como una causa del problema público, resulta parcialmente verdadero, en tanto estas unidades organizativas son meras coordinadoras de los procesos de contratación, que realizan procesos de compras a expensas de los funcionarios responsables del manejo presupuestario, en particular el jerarca de los entes públicos.

Déficit de formulación de la política pública

Como causas del problema público, no fueron consideradas los siguientes aspectos:

i. Interés de las grandes empresas de continuar acaparando las compras del Estado, lo que resulta lógico, en tanto la empresa privada es movida por el interés particular, que se materializa en una mayor ganancia económica, lo que es legítimo, de conformidad con el mercado, y al estilo nacional de desarrollo que había imperado en el país, fundamentado en este.

ii. Las empresas Mipymes no cuentan con los recursos financieros para hacer frente a los gastos financieros en que se debe incurrir en los procesos de contratación en el Estado, como son las garantías, en el caso de Costa Rica, de participación y cumplimiento, tiempos de espera de 30 a 45 días naturales para que se realicen los pagos por parte de los entes públicos o por los costos relacionados con inscripción en el registro de proveedores, la preparación de ofertas y la suscripción de contratos ${ }^{41}{ }^{42}$. Aunque se menciona levemente, como

\footnotetext{
39 Cegesti, Análisis de la realidad...

40 Christian Campos Monge, «Compras de Gobierno y las Pymes participantes en proceso de articulación productiva», ICAP, Revista Centroamericana de Administración Pública, n. 65 (2013): 213-232.

41 Cegesti, Análisis de la realidad...

42 Campos Monge, «Compras de Gobierno...».
} 
una posible causa de no participación en las compras públicas, no se le brinda respuesta en la política.

iii. El ámbito normativo no cuenta con reglas que permita un mayor acceso de las Pymes a las compras públicas, solo existe la posibilidad, en el artículo 20 de la Ley de Fortalecimiento de las Pequeñas y Medianas Empresas, que, en caso de empate, se escoja a la empresa Pymes ${ }^{43} 44$.

iv. La política pública de la administración Chinchilla Miranda entonces contenía un déficit de formulación, en tanto la hipótesis causal, no consideró como causas las ya mencionadas y, por tanto, la hipótesis de solución no comprendería esas causas no identificadas.

Solución al problema público que pierde fuerza

La licitación en estudio concluye su proceso de contratación en la primera mitad de la administración Solís Rivera, administración que había dejado sin efecto la política pública de la administración Chinchilla Miranda mediante la oficialización de la «Política de Fomento al Emprendimiento de Costa Rica», la cual deja por fuera el tema de las compras públicas por parte del Estado a las Mipymes ${ }^{45}$.

La democratización de las compras públicas fue parte de una política pública que muere con la administración Chinchilla Miranda, pero debe implementarse -al menos para el caso seleccionado para estudio- en la administración Solís Rivera, con el agravante de que Chinchilla llega al poder por el Partido Liberación Nacional (PLN) y Solís Rivera por el Partido Acción Democrática (PAC). Aunque ya no existía la política pública para fomentar las compras públicas en la administración Solís Rivera, la institucionalidad del país obligó a esta última administración a continuar con el caso de estudio, en tanto existía un presupuesto anual asignado para tal fin y se había iniciado con el proceso de contratación.

También, sucede un hecho coyuntural específico que incidió en la licitación en estudio y es que la administración Solís Rivera inició el 8 de mayo de 2014, con la noticia que cerraría el Conavi, de conformidad con su promesa de campaña, que era la institución pública responsable del proceso de contratación. Esto generó reacciones adversas de los funcionarios de la institución a los que, en la política pública en la administración anterior se había tratado de motivar y concientizar para que fuera posible las compras públicas con participación de Pymes. Finalmente, la institución no se cerró.

43 Cegesti, Análisis de la realidad...

44 Campos Monge, «Compras de Gobierno...».

45 Contraloría General de la República, expediente de Licitación Pública... 
Posible solución a la democratización de los contratos de conservación y mantenimiento vial

Con la creación del Conavi en 1998, la construcción, conservación y mantenimiento de la red vial nacional del país estaría a cargo de las empresas privadas, contratadas para ese fin. El Estado, en materia de conservación y mantenimiento mediante el Conavi, contrataría empresas privadas que periódicamente brindarían conservación y mantenimiento vial a las 22 zonas en las que había dividido el país para tal efecto. Durante los primeros 16 años, estas contrataciones fueron asumidas por las grandes empresas constructoras nacionales del país, entre ellas Meco S.A., Santa Fe S.A., H. Solís y Sánchez Carvajal.

El objeto de esas contrataciones incluía el mantenimiento y la conservación de la capa asfáltica, la señalización de estas y el despeje de la vegetación, escombros y similares en los alrededores de las carreteras.

En lo referente a la labor de la capa asfáltica, se requería de maquinaria y herramientas especializadas y recurso humano especializado, lo que conlleva a una gran inversión. Para el caso de la señalización los requerimientos son menores, y para las labores de limpieza de vegetación y escombros se requiere una inversión mucho menor.

Con el propósito de democratizar las compras del Estado, para que las Mipymes tuvieran acceso, en la administración Chinchilla Miranda se decide, dentro del marco de «Política Pública de Fomento a las Pyme y al Emprendedurismo»46, licitar en forma separada los componentes de limpieza de vegetación, escombros y similares de la red vial nacional, lo que popularmente se conoce en el país como «chapea», y no considerar dicho objeto contractual entre las contrataciones tradicionales de conservación y mantenimiento del mercado cautivo de las grandes empresas constructoras.

Entre 1998 y 2014, las contrataciones de conservación y mantenimiento vial, además del trabajo especializado relacionados con vías y pasos de agua, debieron realizar la limpieza de hierbas y basura de la red vial nacional. Esto último fue parte de las contrataciones en mención, subcontratando las grandes empresas a microempresas o a grupos de peones (operarios de campo) para realizar esa labor. La licitación promovida en la administración Chinchilla Miranda separó esas labores no especializadas con el propósito de que el Estado contratara directamente a Mipymes, y no por medio de la subcontratación que realizaban las grandes empresas. Esto implicaría la pérdida de beneficios económicos para las grandes empresas, en beneficio de las Mipymes, especialmente los de la zona en que se llevarían a cabo las labores.

46 Ibíd. 
Amplia difusión e información de los entes públicos

Entre 2013 y 2104 , el MEIC ${ }^{47}$, el INA y el Conavi realizaron esfuerzos conjuntos para informar a las Pymes del país de lo concerniente a contratación; así mismo se les brindó capacitación en los procesos de compras públicas. El despliegue informativo incluyó publicaciones en los diarios nacionales de mayor circulación (La Nación, La República, La Prensa Libre, La Teja y Diario Extra), así como comunicados en el sitio web del Conavi y del MEIC. La capacitación se brindó por medio del INA, con cursos diseñados para dicho grupo de empresarios.

En el 2014, fue promovida la Licitación Pública No. 2014LN-000016-0CV00 MR-I: Mantenimiento Rutinario sin Maquinaria Especializada de la Red Vial Nacional Pavimentada, para que en las 22 zonas de la red vial nacional se brindarán los siguientes servicios ${ }^{48}$ :

i. Limpieza de tomas, cabezales y alcantarillas.

ii. Limpieza de cunetas y canales revestidos de manera manual.

iii. Chapea del derecho de vía.

iv. Recolección de basura.

v. Descuaje de árboles por hora.

vi. Brigada de limpieza de puentes.

vii. Aplicación química en malezas y activos viales.

Previo al recibo de ofertas, el MEIC y el Conavi realizaron lo siguiente:

i. Entre el 17 y 31 de octubre de 2014, un borrador del cartel fue sometido a consulta previa.

ii. Del 28 de noviembre al 02 de diciembre de 2014, fue suministrado un borrador del cartel, que había superado la etapa anterior de consulta previa.

iii. El 2 de diciembre de 2014, en el auditorio del MEIC, se llevó a cabo una audiencia que abría un espacio de diálogo para las Pymes, en que se trataron temas del cartel y la factibilidad de ofertar, entre otros.

Durante el 2014 y el 2015, los interesados solicitaron información al Conavi, cuyos funcionarios en forma adicional, aclararon dudas sobre la contratación por 
diversos medios (llamadas telefónicas, en forma presencial, por correo electrónico o por correspondencia tradicional).

La fecha para recibir ofertas fue redefinida en varias ocasiones desde diciembre de 2015 hasta junio de 2016, como producto por parte de los interesados, de solicitudes de aclaración al cartel, recursos de objeción al cartel y diversas peticiones.

Estas actividades clave sucedieron en la administración Solís Rivera, que no había impulsado la democratización de las compras públicas, como sí lo había hecho la administración Chinchilla Miranda, por lo que la contratación ya no gozaba del impulso que se había dado desde la dirección superior del Poder Ejecutivo y quedó en manos de la burocracia del Conavi.

\section{Requisito de admisibilidad}

Al analizar el cartel final (términos cartelarios)49 50 , en su apartado de admisibilidad, es decir, las condiciones mínimas que se deben cumplir para ser considerado como posible contratista, se observa que estas son totalmente abiertas, lo que permite una gran puerta por donde pueden transitar todos los empresarios, no se toman en cuenta aspectos como experiencia y proximidad con el lugar en que se realizan las obras.

En la administración Chinchilla Miranda, la administración del Conavi en asocio con el MEIC, había considerado incluir como requisito de admisibilidad la experiencia en las obras o similares, pero la burocracia del Conavi en la administración Solís Rivera no sostuvo dicha justificación, ante el cuestionamiento hecho por diferentes empresarios con los recursos de objeción al cartel presentados a la Contraloría General de la República (CGR), el ente que en definitiva resuelve sobre ese recurso.

Entre la burocracia del Conavi no existía la motivación para hacer una defensa de un proyecto que había iniciado en la administración anterior, al que se debió ejecutar la contratación. Si el propósito era abrir las posibilidades a los pequeños empresarios que durante muchos años se han dedicado a esa labor, subcontratados por las grandes empresas constructores, este era un aspecto de suma importancia que debía considerarse, la institucionalidad de las compras públicas en Costa Rica, permite el detalle, no solo del objeto contractual, sino de los requisitos de admisibilidad relacionados con ello.

Finalmente, luego del proceso anteriormente indicado, tres fueron ${ }^{51}$ los requisitos por evaluar para la selección de la empresa, según la tabla siguiente.

49 Ibíd.

50 Ledezma Rodríguez, «Un planteamiento de mecanismos...».

51 Contraloría General de la República, expediente de Licitación Pública... 
Tabla 1

Factores por considerar en la Licitación Pública No. 2014LN-000016-0CV00 MR-I: Mantenimiento Rutinario Sin Maquinaria Especializada de la Red Vial Nacional Pavimentada

Factor por evaluar

Puntos

Precio (se evaluará el precio conforme a los apartados

85 anteriores)

Pyme (se debe presentar la certificación Pyme oficial del

10

MEIC según Ley 8262)

Empresa regional (su domicilio se ubica dentro de la línea

5

que ofertará, la cual debe certificarse mediante una institución pública competente)

Total

Fuente: Dirección de Proveeduría del Conavi, 2019.

El principal factor por considerar fue el precio, en tanto representaba el $85 \%$ del criterio para evaluar, un porcentaje de esa magnitud disminuiría por sí mismo los otros dos factores, para efectos de la adjudicación.

El aspecto de menor precio sería el domicilio de la empresa, que estuviera en la zona objeto de contratación (eran 22 zonas), lo que es muy cuestionable, en tanto se considera el domicilio legal, no las instalaciones físicas.

El único aspecto, medianamente rescatable para el fin que se requería alcanzar, es decir, la democratización de las compras públicas en un mercado cautivo por los grandes empresarios, es el criterio de la inscripción como empresa Pyme, que representaba apenas un $10 \%$ del aspecto por considerar, pero el mismo resultó deficiente, como en líneas posteriores se analiza.

Aunada al momento en que se realiza la contratación, es decir en la administración siguiente, a la que patrocinaba, con un burocracia no motivada con el objeto de la democratización de las compras públicas, es necesario considerar que los pequeños y medianos empresarios no mostrarían un papel activo en la redefinición de los elementos de admisibilidad y selectividad para la contratación, lo cual obedece a que no contaban con las competencias necesarias en compras públicas ni tuvieron los recursos financieros para contratar a asesores en la materia que pudieran orientarlos en ese objetivo. 
Para el 2016, el Conavi presupuestó 1000 millones de colones para hacer frente a los pagos que debían realizarse inicialmente a las empresas contratadas, para el objeto contractual en mención.

\section{Adjudicación inicial}

La licitación comprendía la contratación de servicios de «chapea» para las 22 zonas en que se encuentra dividido el país, para efectos de conservación y mantenimiento vial, para un total de 7786 kilómetros de carreteras viales nacionales, en un país que tiene $51179 \mathrm{~km}^{2}$.

En la administración Solís Rivera, el 14 de diciembre de 2016, el Conavi adjudicó 19 de las 22 líneas licitadas a tres empresas relacionadas con la compañía $\mathrm{H}$. Solís, una de las mayores empresas del país, en el área de la construcción de carreteras nacionales ${ }^{52}$.

El monto contratado llegaba en esa ocasión a un poco más de 14 mil millones de colones, que quedaron repartidos entre socios de familiares de la empresa y de uno de sus ingenieros residentes, que formaron empresas para participar en dicho proceso ${ }^{53}$.

Las restantes tres líneas las ganaron la firma Geoastec-Impasesa y dos oferentes particulares: Cesar Jiménez Cubero y Olivier Cubero Arias, los tres son Pymes ${ }^{54}$.

Del total de regiones del país, únicamente el $13 \%$ de ellas había sido adjudicada a Pymes, según era el objetivo de la política de la administración Chinchilla Miranda, y el $87 \%$ de las regiones, contrario a la intensión de la licitación, continuaban siendo contratadas por empresas hijas de una de las mayores empresas de Costa Rica en el área de construcción.

Sobre dicha adjudicación, una de las autoridades del Ministerio de Obras Públicas y Transportes indicó lo siguiente:

No era lo deseable, nos hubiera gustado que pequeñas iniciativas locales o cuadrillas de grupos familiares que se organizaran y participaran y resultaran adjudicatarias. Lo que pasa es que lamentablemente eso sucede en este gremio y cualquier otro. Las empresas grandes tienen, además del poderío económico, todo el acompañamiento legal para apoyar a sus familiares en la formulación de sus propias empresas. Entonces yo diría que el objetivo (de democratizar las licitaciones) se cumplió parcialmente. ${ }^{55}$

Esta adjudicación inicial en favor de los grandes empresarios, que no se sostuvo en la adjudicación final en la proporción señalada como se verá en posteriores

\footnotetext{
52 Álvaro Murillo, «Familiares de mega constructora H. Solís acapararon contratos para Pymes», Semanario Universidad, semana del 18 al 24 de mayo de 2016, acceso: 12 de mayo de 2019, https:// semanariouniversidad.com/pais/familiares-megaconstructora-h-solis-acapararon-contratos-pymes/

53 Ledezma Rodríguez, «Un planteamiento de mecanismos...», 6.

54 Ibíd.

55 Alfaro, citada por Murillo.
} 
líneas, demuestra el interés del gran empresario de seguir beneficiándose con las compras del Estado y desplegar esfuerzos para no permitir el acceso a las medianas y pequeñas empresas.

\section{Adjudicación final}

La institucionalidad del país permitió que varias de las empresas interesadas apelaran el resultado de la adjudicación ante la CGR, proceso que fue llevado a cabo principalmente en el 2016.

Como se deriva del expediente de la Licitación Pública No. 2014LN-0000160CV00 MR-I: Mantenimiento Rutinario sin Maquinaria Especializada de la Red Vial Nacional Pavimentada, existió una feroz lucha, en la vía administrativa, por parte de las empresas relacionadas con la megaempresa $\mathrm{H}$. Solís para no perder la adjudicación en ninguna de las zonas que había participado.

Luego de finalizar el proceso de apelaciones, no fue posible adjudicar la zona Alajuela-Norte, ni la de Pérez Zeledón, y casi el 29 \% del total adjudicado quedó en manos de Fresa Fresca S. A. y Also Frutales S. A. vinculadas a una de las grandes empresas del país, que tienen cautivo el mercado nacional, quedando el $71 \%$ del total adjudicado en manos de los pequeños y medianos empresarios. Es necesario anotar que a un consorcio formado por dos empresas (Ecoterra y Transecot) también le fue adjudicada con un 15,33 \% de la licitación, que forman parte de ese $71 \%$ indicado.

Lo que permitió revertir parcialmente el proceso de adjudicación fue principalmente (no exclusivo) el factor del $10 \%$ de inscripción de la empresa Pymes en el MEIC, ya que permitió el cuestionamiento de las empresas vinculadas a los grandes empresarios en actividades Pymes ${ }^{56}$, relacionadas con lo que popularmente se denomina chapeo. De esta forma, una de las empresas relacionadas al gran capital presentó una certificación de Pymes vinculadas al comercio y no a los servicios, que era lo que se requería, lo que permitió posteriormente en el proceso de resolución de las adjudicaciones, anular la adjudicación que inicialmente se le había hecho. La adjudicación final es la que se indica en la tabla siguiente. 


\section{Tabla 2}

Monto adjudicado por empresa en el proceso de Licitación Pública No. 2014LN-000016-0CV00 MR-I: Mantenimiento Rutinario Sin Maquinaria Especializada de la Red Vial Nacional Pavimentada, 2020.

\begin{tabular}{|c|c|c|c|}
\hline Adjudicado & Línea/zona & Monto & $\begin{array}{l}\text { Porcen- } \\
\text { taje }\end{array}$ \\
\hline \multirow{4}{*}{$\begin{array}{l}\text { Also Frutales } \\
\text { S.A. }\end{array}$} & Línea 11: 2-2 Cañas & $\mathbb{L} 562.690 .481$ & \multirow[t]{4}{*}{18,73} \\
\hline & Línea 6: 1-6 San Ramón & $\mathbb{\Perp} 718.669 .711$ & \\
\hline & Línea 9: 1-9 Heredia & $\mathbb{L} 666.214 .110$ & \\
\hline & Línea 10: 2-1 Liberia & $\mathbb{L} 711.806 .438$ & \\
\hline \multirow{3}{*}{$\begin{array}{l}\text { Consorcio } \\
\text { Ecoterra - } \\
\text { Transecot }\end{array}$} & Línea 1: 1-1 San José & $\mathbb{L} 863.819 .400$ & \multirow[t]{3}{*}{15,33} \\
\hline & Línea 20: 5-2 Limón & $\mathbb{L} 736.363 .500$ & \\
\hline & Línea 22: 6-2 Los Chiles & $\mathbb{L} 577.319 .700$ & \\
\hline \multirow[t]{2}{*}{$\begin{array}{l}\text { Fresa Fresca } \\
\text { S.A. }\end{array}$} & $\begin{array}{l}\text { Línea 12: 2-3 Santa } \\
\text { Cruz }\end{array}$ & $\mathbb{1 6 9 9 . 3 9 9 . 2 6 6}$ & \multirow[t]{2}{*}{10,03} \\
\hline & Línea 13: 2-4 Nicoya & $\mathbb{1} 725.609 .240$ & \\
\hline $\begin{array}{l}\text { Constructora } \\
\text { Mary José, } \\
\text { S.A. }\end{array}$ & Línea 19: 5-1 Guápiles & $\not 938.348 .300$ & 6,61 \\
\hline $\begin{array}{l}\text { Contraolasa } \\
\text { S.A. }\end{array}$ & Línea 7: 1-7 Cartago & $\mathbb{1} 937.701 .435$ & 6,60 \\
\hline $\begin{array}{l}\text { Juan Carlos } \\
\text { Cubero Arias }\end{array}$ & Línea 4: 1-4 Alajuela & $\begin{array}{l}\mathbb{L} 808.086 .487, \\
80\end{array}$ & 5,59 \\
\hline $\begin{array}{l}\text { Adrián Solano } \\
\text { Rodríguez }\end{array}$ & $\begin{array}{l}\text { Línea 21: 6-1 San } \\
\text { Carlos }\end{array}$ & $\mathbb{1} 738.003 .090$ & 5,20 \\
\hline
\end{tabular}




\begin{tabular}{|c|c|c|c|}
\hline Adjudicado & Línea/zona & Monto & $\begin{array}{l}\text { Porcen- } \\
\text { taje }\end{array}$ \\
\hline $\begin{array}{l}\text { Trabajos y } \\
\text { Mantenimiento } \\
\text { Trejos, S.A. }\end{array}$ & Línea 18: 4-3 Sur Sur & $\not 6673.169 .136$ & 4,74 \\
\hline $\begin{array}{l}\text { César Jiménez } \\
\text { Cubero }\end{array}$ & Línea 15: 3-2 Quepos & $\begin{array}{l}\mathbb{L} 662.934 .296, \\
08\end{array}$ & 4,67 \\
\hline $\begin{array}{l}\text { Olivier Cubero } \\
\text { Arias }\end{array}$ & $\begin{array}{l}\text { Línea 14: 3-1 } \\
\text { Puntarenas }\end{array}$ & $\not 608.977 .103$ & 4,29 \\
\hline $\begin{array}{l}\text { Albin Gerardo } \\
\text { Thames Rojas }\end{array}$ & Línea 8: 1-8 Turrialba & 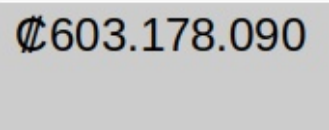 & 4,25 \\
\hline $\begin{array}{l}3-101-553488 \\
\text { S.A. }\end{array}$ & Línea 2: 1-2 Puriscal & $\not \mathbb{L} 599.684 .999$ & 4,22 \\
\hline $\begin{array}{l}\text { Inversiones } \\
\text { Solano y } \\
\text { Camacho, S.A. }\end{array}$ & Línea 3: 1-3 Los Santos & 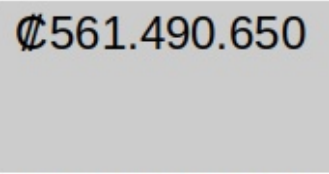 & 3,95 \\
\hline \multirow[t]{2}{*}{$\begin{array}{l}\text { Ana Lorena } \\
\text { Chaves Rivera }\end{array}$} & $\begin{array}{l}\text { Línea 17: 4-2 Buenos } \\
\text { Aire }\end{array}$ & $\mathbb{C} 807.046 .285$ & 5,68 \\
\hline & & $\begin{array}{l}\mathbb{L} 14.200 .511 .7 \\
17,88\end{array}$ & 99,89 \\
\hline
\end{tabular}

Fuente: Dirección de Proveeduría del Conavi, 2019.

Grandes excluidos

Los peones que forman cuadrillas (grupo de peones), dedicados por años a la limpieza de hierbas y basura de la red vial nacional, fueron radicalmente excluidos de la Licitación Pública No. 2014LN-000016-0CV00 MR-I: Mantenimiento Rutinario sin Maquinaria Especializada de la Red Vial Nacional Pavimentada, en tanto fueron invisibilizados en la política pública de la administración Chinchilla Miranda en las acciones de esa administración y en la administración Solís Rivera ni siquiera mencionados.

Existe un reconocimiento implícito en el cartel, el expediente de la contratación, así como en las acciones, que el trabajo de limpieza de hierbas y basura es llevado a cabo por grupos de peones sin maquinaria especializada, es decir, donde priva principalmente su fuerza de trabajo, son ellos los que realizan directamente los trabajos. Estos peones no se encuentran organizados en empresas de ningún tipo y, para ellos, las compras públicas son procesos ajenos a su participación. 
La licitación fue dirigida principalmente a los pequeños y medianos empresarios, dejando incluso en forma marginal al microempresario, que para el caso de Costa Rica, según la clasificación brindada por la Caja Costarricense de Seguro Social (CCSS), son aquellas empresas que tienen 5 o menos empleados, excluyendo totalmente a los trabajadores no organizados.

Entre las empresas que finalmente fueron adjudicadas, hay pequeñas y medianas empresas, además de las vinculadas a una de las grandes empresas nacionales que no se encuentran formadas por cuadrillas de peones, sino en algunos casos son profesionales o comerciantes que formaron una empresa que participó en la licitación.

En el periodo de análisis, no existieron iniciativas que fructificaran para agrupar a estos trabajadores (peones) en empresas autogestionarias (donde fueran trabajadores y propietarios al mismo tiempo) para que participarán en la licitación, lo que hubiera llevado un trabajo de mayor profundidad por parte de las burocracias del Conavi, INA y MEIC; así, iniciativas como la formación de cooperativas autogestionarias que agruparan a esos peones para posteriormente capacitarlos, asesorarlos y hasta brindar respaldo financiero, para que fueran los adjudicados, fueron fallidas.

Al 31 de diciembre de 2021, las autoridades del Conavi no se encontraban considerando la continuidad y mejora de la experiencia de pretender democratizar los contratos de conservación y mantenimiento vial, es decir no tenían considerado promover otra licitación con el mismo objeto contractual, de ser así, las lecciones aprendidas de la licitación analizada, no se aprovecharían, pero peor aún, seguirían sin democratizarse las compras públicas.

\section{Causas}

Las causas por las que no se logró el objetivo de democratizar la contratación de limpieza de hierbas y basura de la red vial nacional (Licitación Pública No. 2014LN-000016-0CV00 MR-I: Mantenimiento Rutinario sin Maquinaria Especializada de la Red Vial Nacional Pavimentada), fueron las siguientes:

i. La licitación quedó sin patrocinador debido a que fue impulsada en la administración Chinchilla Miranda (2010-2014) como parte de «Política Pública de Fomento a las Pyme y al emprendedurismo», pero el concurso fue llevado a cabo en los dos primeros años de la administración Solís Rivera (2014-2018), en la que la política no se encontraba vigente.

ii. Al perder el patrocinador, la licitación quedó en manos de la burocracia del Conavi de la administración Solís Rivera, que de conformidad con las motivaciones propias del homus burocratics, no se encontraba interesada en el objetivo de la democratización de las compras públicas, siendo en ese momento su preocupación principal conservar el empleo ante el anuncio y acciones de la administración Solís Rivera de cerrar el Conavi, que era la institución responsable del concurso. 
iii. La negativa del gran empresario de permitir la participación de los micro, pequeños y medianos empresarios en las compras públicas, siendo su interés, dentro de la naturaleza del homus economics, el seguir lucrando de ella, lo que es legítimo y legal en el actual estilo nacional de desarrollo, dada su institucionalidad.

iv. No existe una normativa, en Costa Rica, como sí existe en otros países que, mediante mecanismos efectivos, fomente las compras públicas con participación de pequeños y medianos empresarios.

v. Los pequeños y medianos empresarios no cuentan con los conocimientos suficientes para participar en forma efectiva de procesos de compras públicas ni con el capital financiero para hacer frente a los compromisos financieros que implican los procesos de compras públicas.

vi. Los pequeños y medianos empresarios no cuentan con asesoría efectiva en todo el proceso de contratación porque el Estado, o alguna organización de la sociedad civil, no lo brinda ni dichas empresas tienen el capital económico suficiente para contratar.

vii. Los peones, o grupos de ellos (que son los que realizan directamente el trabajo de limpieza), fueron invisibilizados en todo el proceso, lo que inició con su exclusión como actores objetivos de la licitación. Solo esto demuestra que existió en la presente licitación, un proceso auténtico de democratizar la compra pública.

\section{Recomendaciones}

\section{A nivel general}

i. Crear el marco normativo que permita a las micro, pequeñas y medianas empresas, especialmente a las autogestionarias, contratar con el Estado, como podría ser cuotas de compras públicas para Mipymes, la tolerancia de un precio relativamente mayor o la contratación de grupos de peones con experiencia en el servicio.

ii. Fomentar la preparación para que los micro, pequeños y medianos empresarios, especialmente los autogestionarios, puedan participar en el proceso de compras públicas del Estado.

iii. Fomentar, por parte del Estado, la creación de empresas autogestionarias, entre ellas las cooperativas autogestionarias, que permitan agrupar a los peones que realizan directamente las labores y darles formación y acompañamiento en el proceso de contratación. 
iv. Realizar estudios para que puedan redefinirse los objetos contractuales de las compras públicas, acaparadas por las grandes empresas, para que las mismas sean de acceso a los micro, pequeños y medianos empresarios, especialmente a los autogestionarios.

\section{Con el caso concreto}

i. Con el propósito de mantener el rumbo de la democratización de las compras públicas, al vencimiento de los contratos producto de licitación No. 2014LN-000016-0CV00 MR-I (caso en análisis), se pueda realizar nuevamente la contratación, con las lecciones aprendidas, tanto en la contratación como en la ejecución, y con un acompañamiento puntual en cada uno de los aspectos clave del proceso de contratación (inscripción de proveedores, presentación de ofertas, presentación de garantías de participación y cumplimiento).

ii. Organizar y capacitar a los trabajadores (peones) de las localidades en que se realizan los trabajos de chapea (limpieza) para que puedan ser objeto de adjudicación de compras públicas que se realicen en sus comunidades.

\section{Formato de citación según APA}

Vásquez-Rodríguez, J. A. (2021). La democratización de los contratos de conservación y mantenimiento de la red vial nacional: Un caso fallido en Costa Rica. Revista Espiga, 21(42), 220-247.

\section{Formato de citación según Chicago-Deusto}

Vásquez-Rodríguez, Jorge Alberto. «La democratización de los contratos de conservación y mantenimiento de la red vial nacional: Un caso fallido en Costa Rica». Revista Espiga 21, n.o 42 (julio-diciembre, 2021): 220-247. 


\section{Referencias}

Berelson, Bernard. Content Analysis in Communication Researches. United States of America: Free Press, 1952.

Campos Monge, Christian. «Compras de Gobierno y las Pymes participantes en proceso de articulación productiva». ICAP, Revista Centroamericana de Administración Pública, n. ${ }^{\circ} 65$ (2013): 213-232.

Cegesti. Análisis de la realidad de acceso y participación de las Pymes en las compras públicas en Costa Rica. Programa ICT4GP, Ministerio Relaciones Exteriores Reino de los Países Bajos. Costa Rica, 2012. Acceso: 12 de octubre de 2020. http://comprasresponsables.cegesti.org/articulos/ articulo-25-260213_informe_compras_publicas_PYME_CR.pdf

Contraloría General de la República. Expediente de Licitación Pública No. 2014LN-000016-0CV00 MR-I: Mantenimiento Rutinario Sin Maquinaria Especializada de la Red Vial Nacional Pavimentada. Contraloría General de la República, Costa Rica, 2015. Acceso: 21 de noviembre de 2019. https:// cgrweb.cgr.go.cr/pr02/f?

$p=307: 135::: N O: 135: P 135$ COD_ENTIDAD,P135_COD_UNIDAD_CONTRATAN TE,P135_NUM_PROCEDIMIĒNTO,P135_TIPO_PROCCEDIMIENTO:2100042008, $100000 \overline{0} 415, \overline{2} 014 \mathrm{LN}-000016-0 \mathrm{CV} 00, \overline{0} 1$

Cunill, Nuria. «¿Qué ha pasado con lo público en los últimos 30 años? Balance y perspectivas». Revista del CLAD Reforma y Democracia, 5-7, 2012.

Gallardo, Helio. Fundamentos de Formación Política. Análisis de Coyuntura. Costa Rica: Red Alforja Roja, 1990.

Ledezma Rodríguez, Yesenia. «Un planteamiento de mecanismos para la evaluación de las ofertas que fomenten en las Pymes mayor accesibilidad de las compras de la administración pública en Costa Rica». Tesis de Maestría en Gestión de la Innovación Tecnológica, Universidad Nacional, Costa Rica, 2015.

López Noguero, Fernando. «El Análisis de Contenido Como Método de Investigación». Revista de Educación, 4 (2002): 167-179, Universidad de Huelva, 2002.

Merton, Robert. Teoría y estructura sociales. México: FCE, 1964.

Murillo, Álvaro. «Familiares de mega constructora H. Solís acapararon contratos para Pymes». Semanario Universidad. Semana del 18 al 24 de mayo de 2016. Acceso: 12 de mayo de 2019. https://semanariouniversidad.com/ pais/familiares-megaconstructora-h-solis-acapararon-contratos-pymes/ 
Márquez Blas, Robert. «Teorías clásicas de la burocracia capitalista: Hegel, Marx, Weber y Michells». Revista de Colaboración Jurídica, n. 76 (1990): 77-89.

Rovira Mas, Jorge. «Costa Rica: El actual estilo nacional de desarrollo y sus principales tendencias», en Róger Churnside y Eduardo Lizano (Compiladores y editores), Sociedad para el Avance de la Socioeconomía (SASE). Selecciones de la XX Conferencia Costa Rica 2008. San José: Academia de Centroamérica, 2009, 163-178. http://www.hacienda.go.cr/ centro/datos/Libro/

Sociedad\%20para\%20el\%20avance\%20de\%20la\%20socioeconom\%C3\%A Da.pdf

Subirats, Joan, Peter Knoepfel, Corinne Larrue y Fréderic Varones. Análisis y gestión de políticas públicas. España: Editorial Ariel, 2008.

Vásquez Rodríguez, Jorge. «La Compleja Institucionalidad de la Infraestructura Vial Nacional en Costa Rica, 1998-2014». Revista Nacional de Administración 8, n. ${ }^{\circ} 1$ (2017): 100-117. https://doi.org/10.22458/ rna.v8i1.1180

Vásquez Rodríguez, Jorge. «La Corporatización Empresarial de las Políticas Pública, el caso de Procomer». Tesis Maestría en Sociología, Universidad de Costa Rica, 2008.

Vásquez Rodríguez, Jorge. «La Gestión Pública en la Encrucijada de lo Público y lo Privado». Costa Rica. Editorial Universidad Estatal a Distancia, 2019.

Vásquez Rodríguez, Jorge. «La Política Pública de la Infraestructura Vial Nacional en Costa Rica (1998-2014)». Tesis doctoral, presentada en el Programa de Doctorado en Gobierno y Políticas Públicas, Universidad de Costa Rica, 2019.

Yin, Robert. Case Study Research: Design and Methods, Applied social research Methods Series. Newbury Park CA, 1989.

Yin, Robert. Applications of case study research. Newbury Park: Sage Publishing, 1993.

Yin, Robert. Applications of Case Study. Thousan Oaks, United States of America: Sage Publishing, 1994.

Zurbriggen, Cristina. «Las redes de políticas públicas. Una revisión teórica». 